that liver-type ALP was produced in a patient with meningioma, but they did not find an abnormal ALP value. In addition, we did not find-abnormal ALP values in 100 patients with non-neoplastic intracranial diseases. Taken together, our data suggest that the abnormal ALP found in the cerebrospinal fluid from this patient was liver-type ALP produced by intracranial cancer cells which had metastasised from an alveolar lung adenocarcinoma.

This work was supported in part by a grant from the Ministry of Education, Science and Culture of Japan.

1 Harris H. The human alkaline phosphatases: what we know and what we don't know. Clin Chim Acta 1990; 186:133-50.

2 Simko V. Alkaline phosphatase in biology and medicine. Dig Dis Sci 1991;9:189-209.

3 Moss DW. The nature and origin of alkaline phosphatase in hepatobiliary disease. $Z$ Med Lab Diagnost 1989;30: 355-63.
4 Alpers DH, Eliakim R, DeSchryver-Kecskemeti $K$. Secretion of hepatic and intestinal alkaline phosphatases: similarities and differences. Clin Chim Acta 1990;186:211-24.

5 Komoda T, Koyama I, Nagata A, Sakagishi Y, Kurata M, Kumegawa M. A possible mechanism of induction and translocation into blood stream of rat alkaline phosphatase activity by bile duct ligation. Arch Biochem Biophys 1986;251:323-35.

6 Miura M, Matsuzaki H, Bailyes EM, et al. Difference between human liver- and bone-type alkaline phosphatases. Clin Chim Acta 1989;168:177-88.

7 Komoda T, Sato M, Furiya K, Sakagishi Y, Sekine T Allelic and ectopic polymorphisms in human placental Allelic and ectopic polymorphisms in human placental alkaline phosphatases: sugar

8 Scallon B, Fung WJ, Tsang C, Li S, Kado-Fong H, Huang KS, Kochan J. Primary structure and functional activity of a phosphatidylinositol-glycan specific phospholipase D. Science 1991;252:446-8.

9 Henderson $A R$, Grace DM. Liver-originating isoenzyme of alkaline phosphatase in the serum: a perioplastic manifestation of a malignant schwannoma of the sciatic nerve. F Clin Pathol 1976;29:237-40.

10 Koyama I, Miura M, Matsuzaki H, Sakagishi Y, Komoda $\mathrm{T}$. Sugar chain heterogeneity of human alkaline phosphatase: differences between normal and tumour associated isozymes. $\mathcal{F}$ Chromatogr 1987;413:65-78.

11 Murakami M, Yoshioka S, Kuratsu J, Nakamura $H$ Ushio Y. High serum alkaline phosphatase level of meningioma cell origin: case report and review of the literature. Neurosurgery 1992;30:624-7.

\title{
Cholecystitis, cholelithiasis, and ganglioneuromatosis of the gall bladder: an unusual presentation of MEN type $2 b$
}

\author{
R Chetty, S P Clark
}

\author{
Department of \\ Anatomical \\ Pathology, The Royal \\ Melbourne Hospital \\ and University of \\ Melbourne, \\ Melbourne, Australia \\ R Chetty \\ S P Clark \\ Correspondence to: \\ Dr R Chetty, \\ Nuffield Department of \\ Pathology, University of \\ Oxford, John Radcliffe \\ Hospital, Headington \\ Oxford OX 3 9DU \\ Accepted for publication \\ 1 June 1993
}

\begin{abstract}
A 40 year old man with multiple endocrine neoplasia type 2b (MEN 2b) presented with cholecystitis caused by gall stones. Twenty four years earlier, he had had a partial thyroidectomy for a cold nodule. At his initial presentation MEN 2b with medullary carcinoma of the thyroid had not been made. This was diagnosed while investigating his gall bladder symptoms and he was found to have asymptomatic residual medullary thyroid carcinoma and bilateral adrenal phaeochromocytomas. The cholecystectomy specimen contained several mixed calculi and extensive ganglioneuromatosis with large, prominent nerves containing ganglion cells in the gall bladder wall.
\end{abstract}

(F Clin Pathol 1993;46:1061-1063)

Neuronal hyperplasia or ganglioneuromatosis are well recognised components of multiple endocrine neoplasia type $2 b(M E N 2 b)^{12}$ and neurofibromatosis. ${ }^{3}$ Ganglioneuromatosis of the gastrointestinal tract often precedes the appearance of medullary thyroid carcinoma and phaeochromocytoma in MEN 2b. ${ }^{4}$ Within the context of MEN $2 b$, presentation related to gall bladder disease is rare. A case of MEN $2 b$ is presented in which the patient complained primarily of symptoms related to gall bladder disease.

\section{Case report}

The patient was a 40 year old man who at the age of 16 years had had a partial thyroidectomy for a cold nodule. This was interpreted to be a Hürthle cell carcinoma at the time. Twenty four years later, he complained of right upper quadrant pain, dyspepsia, and flatulence. Examination showed him to be hypertensive (although he did not complain of hypertension) and to have right upper quadrant tenderness. He was also noted to have a lump in his residual thyroid and bilateral loin masses. A computed tomogram revealed an enlarged gall bladder filled with calculi, a tumour in the residual thyroid, and bilateral adrenal medullary tumours. Subsequent examination showed mucosal neuromas of the lips, tongue, larynx and cornea. Furthermore, the patient was noted to have a marfanoid feature. MEN $2 b$ was diagnosed and a detailed review of the patient's history disclosed a positive family history with the patient's mother having bilateral phaeochro- 
Figure 1 Gall bladder wall thickened by large nerves with a plexiform mucosa is intact and there is mild chronic cholecystitis (haematoxylin and eosin). pattern. The surface

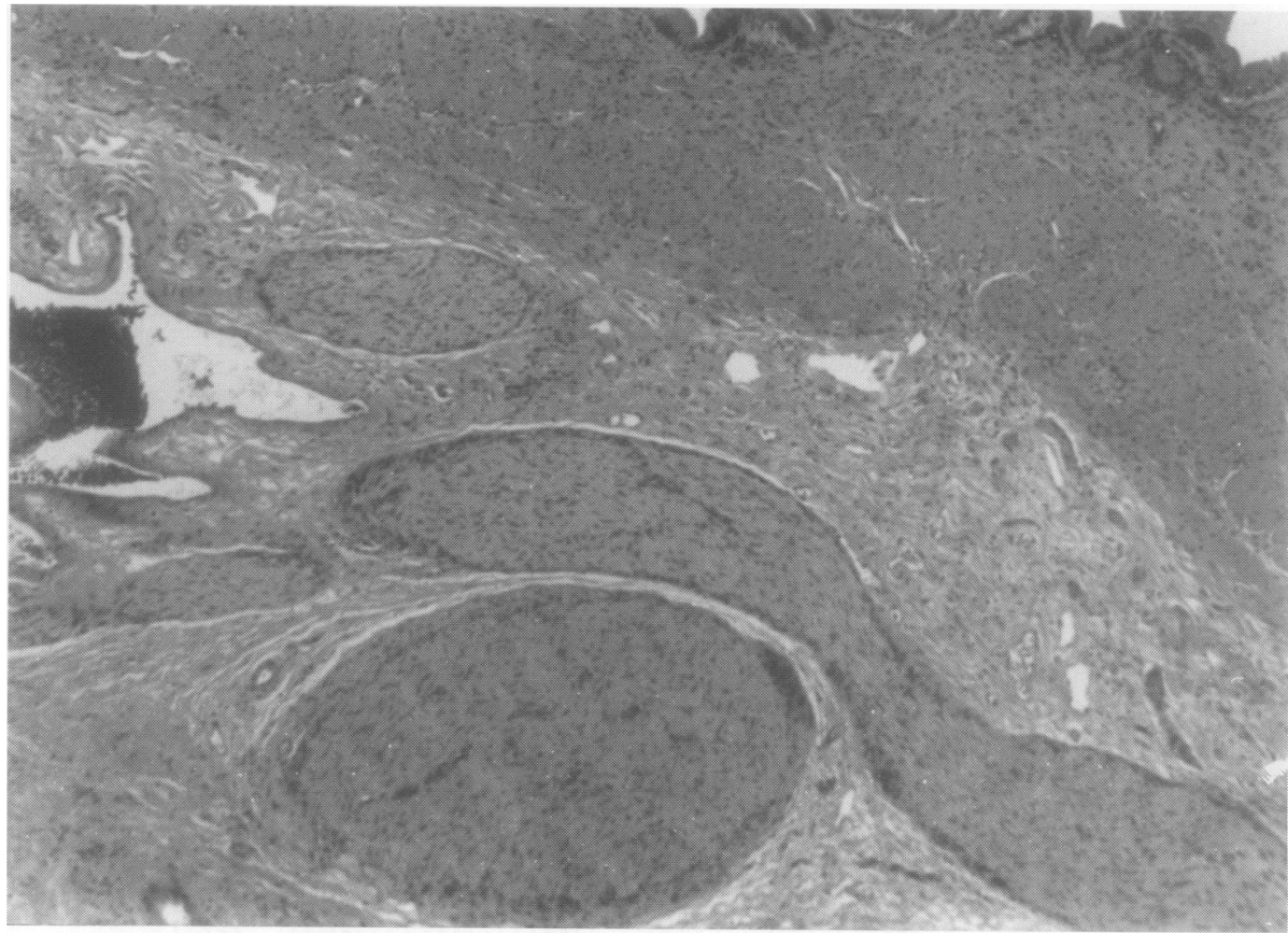

mocytomas. A brother has a similar appearance to the patient and has been treated for hypertension, presumably related to phaeochromocytomas. None of the patient's children had any signs or symptoms of MEN. Serum calcitonin and urinary catecholamine values were increased. The residual thyroid was resected and bilateral adrenalectomy and cholecystectomy were performed. One year later, the patient had symptoms attributable to diverticular disease and a neurogenic bladder.

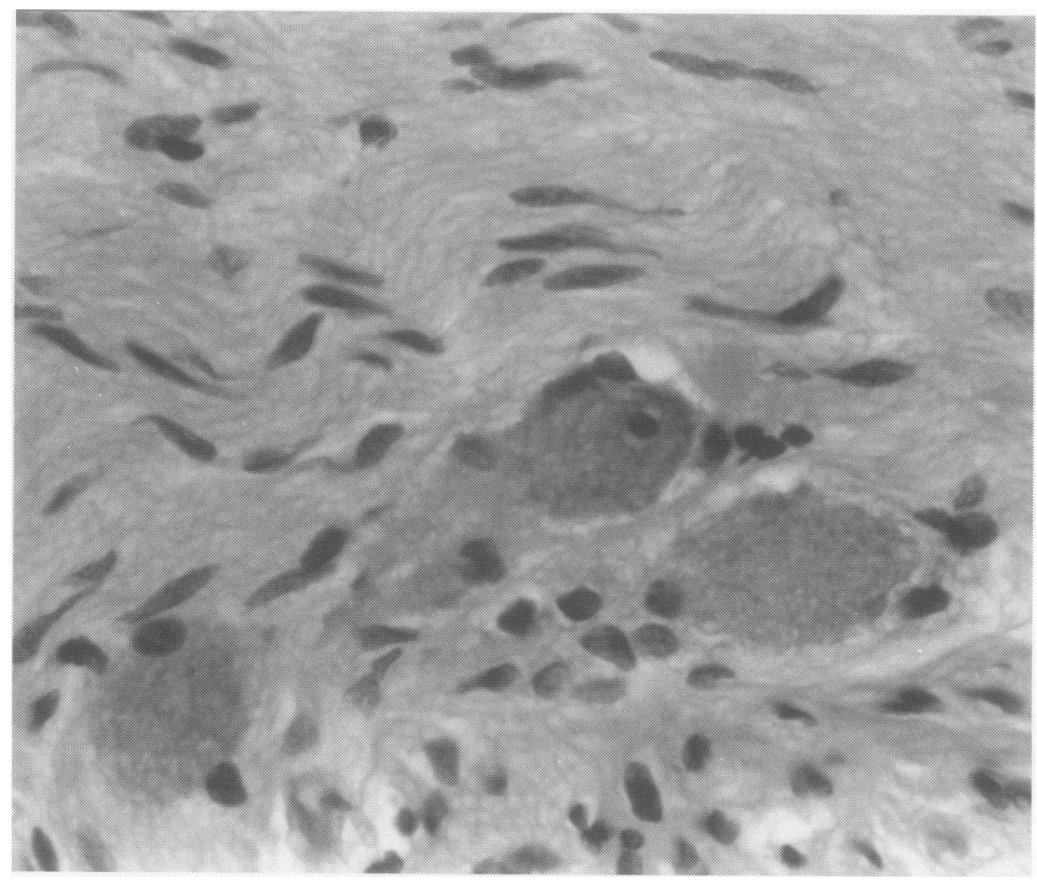

Figure 2 Associated with the neuronal hyperplasia were areas of ganglioneuromatosis with ganglion cells and proliferating Schwann cells (haematoxylin and eosin).

\section{Methods}

All specimens were fixed in $10 \%$ buffered formalin and processed routinely. In addition to haematoxylin and eosin stains, immunohistochemistry was performed using the streptavidin-biotin complex on formalin fixed, paraffin wax embedded tissue for the following antibodies: chromogranin (Boehringer Mannheim, Germany, dilution 1 in 100); synaptophysin (Biogenix Medos, Australia, 1 in 200); S-100 protein (Dakopatts, USA, 1 in 400); neurofilament (Dako, 1 in 50); vasoactive intestinal peptide (VIP) (Biogenix Medos, Australia, prediluted); somatostatin (Dako, 1 in 300 ) and glial fibrillary acidic protein (GFAP) (Dako, 1 in 150).

\section{Pathological findings}

Macroscopically, the gall bladder measured $10 \times 3 \mathrm{~cm}$ and contained several mixed calculi. The wall measured $0.3 \mathrm{~cm}$ in thickness and the serosal surface had a ropy, nodular appearance. The mucosa appeared to be within normal limits. Microscopically, the gall bladder mucosa was intact and lined by normal columnar epithelium (fig 1). The wall deep to the muscle layer and subserosa contained large prominent nerve trunks, some of which were dumbell-shaped, simulating a plexiform neurofibroma (fig 1). The enlarged nerves were accompanied by an increase in the number of ganglion cells embedded within proliferating Schwann cells (ganglioneuromatosis) (fig 2). Occasional "giant ganglia" were also seen. These foci were scattered randomly throughout the gall bladder wall. A mild chronic inflammatory infiltrate with attendant fibrosis was also present. The slides from the first thyroid tumour were reviewed and these, together with the 
current tumour, were typical of medullary carcinomas. Associated areas of C-cell hyperplasia were present. Both adrenal tumours were classic solitary phaeochromocytomas.

The Schwann cells and nerve trunks were strongly positive for S-100 protein and neurofilament. The ganglion cells were focally positive for neurofilament but negative for all other markers.

\section{Discussion}

Ganglioneuromatosis is a consistent finding in MEN 2b. ${ }^{5}$ In fact, one of the most characteristic and evident alimentary tract manifestations of the syndrome is diffuse or nodular enlargement of the lips caused by ganglioneuromatosis. ${ }^{67}$ Major reviews of the gastrointestinal pathology encountered in MEN 2b, however, have only briefly alluded to gall bladder disease. ${ }^{46}$

In his initial review Carney found two patients with gall bladder disease. ${ }^{4}$ One had gall bladder ganglioneuromatosis and the other acalculous cholecystitis. Unfortunately, microscopical examination findings were not available in the latter case. Symptoms related to gall bladder disease are not a common or well documented mode of presentation in MEN 2b.

Ganglioneuromatosis of the gastrointestinal tract causes a plethora of abdominal symptoms, including constipation, diarrhoea, colic, projectile vomiting, and, in the case of infants, difficulty with feeding. Despite these protean manifestations, the usual gastrointestinal symptoms in MEN $2 b$ are intermittent, chronic constipation and chronic diarrhoea. ${ }^{6}$ Ganglioneuromatosis is a well recognised cause of gastrointestinal hypomotility and the associated diarrhoea has been ascribed to high circulating serum calcitonin concentrations produced by medullary carcinoma of the thyroid. ${ }^{6}$ Other peptides, however, may also play a part.

In this case the ganglioneuromatosis of the gall bladder wall might have resulted in poor contraction with a resultant stasis contributing to the formation of the gall stones. It must be remembered, however, that cholelithiasis is common and may be unrelated to the presence of ganglioneuromatosis of the gall bladder.

This case illustrates a very uncommon presentation of a patient with classic MEN $2 b$ who had cholelithiasis and cholecystitis with synchronous, asymptomatic recurrent medullary thyroid carcinoma and bilateral adrenal phaeochromocytomas.

1 Williams ED, Pollock DJ. Multiple mucosal neuromata with endocrine tumours: A syndrome allied to von Recklinghausen's disease. $\mathcal{F}$ Pathol Bacteriol 1966;91: 71-80.

2 Gorlin RJ, Sedano HO, Vickers RA, Cervenka J. Multiple mucosal neuromas, pheochromocytoma and medullary carcinoma of thyroid. A syndrome. Cancer 1968;22. 293-9.

3 Ricardi VM. Von Recklinghausen neurofibromatosis. $N$ Engl f Med 1981;305:1617-27.

4 Carney JA, Go VLW, Sizemore GW, Hayles AB Alimentary tract ganglioneuromatosis: A major component of the syndrome of multiple endocrine neoplasia, nent of the syndrome of multiple endocrine
type 2 b. $N$ Engl f Med 1976;295:1287-91.

5 Fuller CE, Williams GT. Gastrointestinal symptoms of type 1 neurofibromatosis (von Recklinghausen's disease). Histopathology 1991;19:1-11.

6 Carney JA, Hayles AB. Alimentary tract manifestations of multiple endocrine neoplasia, type 2b. Mayo Clin Proc 1977;52:543-8.

7 Carney JA, Sizemore GW, Lovestedt SA. Mucosal ganglioneuromatosis, medullary thyroid carcinoma and pheochromocytoma: multiple endocrine neoplasia, type 2b. Oral Surg 1976;41:739-52. 